\title{
Geometric control of quadrotor UAVs using integral backstepping
}

\author{
Ali Bouchaib ${ }^{1}$, Rachid Taleb ${ }^{2}$, Ahmed Massoum $^{3}$, Saad Mekhilef $^{4}$ \\ ${ }^{1,3}$ Electrical Engineering Department, Djillali Liabes University, Sidi Bel Abbes, Algeria \\ Intelligent Control and Electrical Power Systems Laboratory (ICEPS) \\ ${ }^{2}$ Electrical Engineering Department, Hassiba Benbouali University, Chlef, Algeria \\ Laboratoire Génie Electrique et Energies Renouvelables (LGEER) \\ ${ }^{4}$ Electrical Engineering Department, Malaya University, Kuala Lumpur, Malaysia
}

\section{Article Info \\ Article history: \\ Keywords: \\ Backstepping \\ Geometric \\ Integral \\ Lyapunov \\ Quadrotor}

Received Dec 1, 2020

Revised Feb 14, 2021

Accepted Feb 26, 2021

\begin{abstract}
The traditional quadcopter control systems should deal with two common problems. Namely, the singularities related to the inverse kinematics and the ambiguity linked to the quaternion representation of the dynamic model. Moreover, the stability problem due to the system nonlinearity and high degree of coupling. This paper provides a solution to the two issues by employing a geometrical integral-backstepping control system. The integral terms were added to improve system ability to track desired trajectories. The high-level control laws are considered as a virtual control and transmitted to the low-level to track the high-level commands. The proposed control system along with the quadcopter dynamic model were expressed in the special Euclidean group SE(3). Finally, the control system robustness against mismatching parameters was studied while tracking various paths.
\end{abstract}

This is an open access article under the CC BY-SA license.

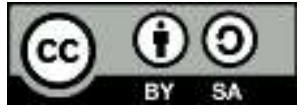

\section{Corresponding Author:}

Ali Bouchaib

Electrical Engineering Department

Djillali Liabes University, Sidi Bel Abbes, Algeria

Intelligent Control and Electrical Power Systems Laboratory (ICEPS)

Email: ali81.bouchaib@gmail.com

\section{INTRODUCTION}

For the last two decades the filed of unmanned aerial vehicles (UAVs) was under rapid growth and many applications were improved by utilizing UAVs (e.g., military inspection, emergency rescue services, and mapping). Because of the intensive research in the filed [1, 2]. Recently, a growing interest in the multirotor UAVs for the systems high capabilities (i.e., vertical take-off, and landing, navigation in narrow spaces and maneuvering capacity) [3-5]. However, the advantages of multi-rotor UAVs comes with highly coupled dynamic models which makes designing a decoupled control law that stabilizes the systems a changing task $[6,7]$. The progress in the domain of multi-rotor UAVs leads to the adoption of geometric modeling to avoid singularities and ambiguity $[8,9]$. However, the nonlinearity and highly coupled systems require an advanced control system. The backstepping control method is considered one of the advanced approaches to regulating nonlinear systems, which can give robust and decoupled control laws [10, 11].

Recently, a new control approach for dynamic systems was developed which is based on nonlinear manifolds that cannot be globally identified with Euclidean spaces. In previous studies [12, 13], a control approach that was designed by characterizing the geometric properties of nonlinear manifolds intrinsically. The geometric control techniques can completely avoid the singularities and ambiguities issues, this approach 
was applied to fully actuated rigid body utilizing mathematical method of Lie groups to achieve almost global asymptotic stability [14-16]. Furthermore, the flying environment's internal and external perturbations do not affect the durability of the mentioned control [17-20].

In this paper, a two-level cascade control system based on integral-backstepping technique was designed to achieve global stability and robustness against mismatching parameters. The low-level is the attitude to adjust the UAVs' attitude angles and maintain a stable flight. The control loop is based on geometric control topology which makes the system able to avoid singularities and commands' ambiguity, then we defined the attitude as a virtual control of the angular dynamic and utilized Lyapunov's theory to ensure system stability.

Moreover, the high-level control system, which is the transitional control loop, we took the linear velocity to be virtual control in this subsystem. First, this control subsystem makes the UAVs reach the desired position. Secondly, it translates the path information to the virtual control inputs of the low-level control system. Finally, the complex trajectories are designed to be the reference input of the system (I cannot understand this).

This paper is organized as follows: in Section 2, the quadrotor dynamic model is introduced. In Section 3, the control system design based on the backstepping geometric method for attitude and integralbackstepping control and stability analysis is presented. Simulation results to prove the controllers' effectiveness are displayed in Section 4. Finally, the conclusions are presented in Section 5.

\section{QUADROTOR DYNAMIC MODEL}

As illustrated in Figure 1, a quadrotor vehicle model is a system of four identical motors and propellers that are located at the vertices of a square and generated thrust and torque normal to the plane of this square. Then, an inertial reference frame $\left\{\overrightarrow{l_{1}}, \overrightarrow{\imath_{2}}, \overrightarrow{l_{3}}\right\}$ and a body-fixed frame $\left\{\overrightarrow{b_{1}}, \overrightarrow{b_{2}}, \overrightarrow{b_{3}}\right\}$ were chosen. Also, the body-fixed frame origin is located at the center of the mass of this vehicle.

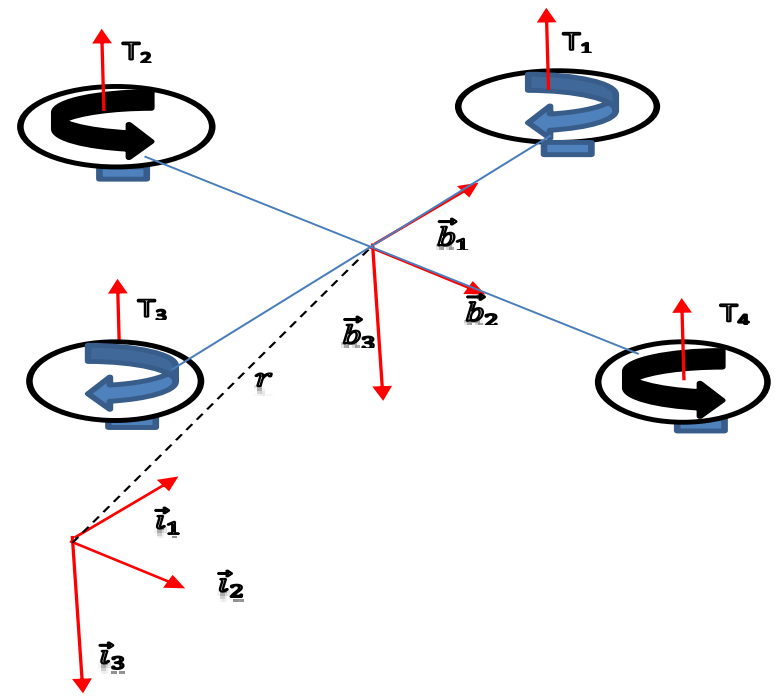

Figure 1. Quadrotor dynamique

The model is represented by the rotation matrix $R \in S O(3)$ [13-16]. Consequently, the configuration manifold is the special euclidean group $\mathrm{SE}(3)$, which is the semi-direct product of $\mathbb{R}^{3}$ and the special orthogonal group $\mathrm{SO}(3)$. The rotation matrix $R$ is given by the product of the three successive matrices: $R=$ $\operatorname{Rot}_{z}(\psi) * \operatorname{Rot}_{y}(\theta) * \operatorname{Rot}_{x}(\theta)$, where $\operatorname{Rot}_{z}(\psi), \operatorname{Rot}_{y}(\theta)$ and $\operatorname{Rot}_{x}(\theta)$ are the partial rotation matrices around $\overrightarrow{b_{3}}, \overrightarrow{b_{2}}$ and $\overrightarrow{b_{1}}$. where $S O(3)=\left\{R \in R^{3 \times 3}: R^{T}=R^{-1} \wedge \operatorname{det}(R)=1\right\}$ [17-20]. The representation of the rotation matrix is depicted in (1) as follow:

$$
R=\left[\begin{array}{ccc}
c \psi c \theta & c \psi s \theta s \phi-s \psi c \phi & c \psi s \theta c \phi+s \psi s \phi \\
c \theta s \psi & s \psi s \theta s \phi-c \psi c \phi & s \psi s \theta c \phi-c \psi s \phi \\
-s \theta & s \phi c \theta & c \phi c \theta
\end{array}\right]
$$


We note that $s \phi$ and $c \phi$ denote $\sin (\phi)$ and $\cos (\phi)$ respectively and similarly for $\theta$ and $\psi$.

The dynamical model of the quadrotor can be described as:

$$
\begin{aligned}
& m \ddot{r}=m g i_{3}-T R i_{3}+F_{\text {ext }} \\
& \dot{R}=R \Omega_{\times} \\
& J \dot{\Omega}+\Omega \times J \Omega=M+M_{\text {ext }}
\end{aligned}
$$

Where the $m$ is a mass and $J$ inertia matrix, $r$ is the quadrotor position in the inertial frame, $\Omega$ is the angular velocity in the body frame, $F_{\text {ext }}$ and $M_{\text {ext }}$ are external force and moment, respectively.

${ }^{\circ} \times \mathbb{R}^{3} \rightarrow S O(3)$ is defined by the condition that $a_{\times} b=a \times b$ for all $a, b \in \mathbb{R}^{3}$

The Euler equation determines the angular acceleration:

$$
\left[\begin{array}{ccc}
J_{x x} & 0 & 0 \\
0 & J_{y y} & 0 \\
0 & 0 & J_{z z}
\end{array}\right]\left[\begin{array}{l}
\dot{\Omega_{1}} \\
\dot{\Omega_{2}} \\
\dot{\Omega_{3}}
\end{array}\right]=\left[\begin{array}{l}
M_{1} \\
M_{2} \\
M_{3}
\end{array}\right]-\left[\begin{array}{c}
\Omega_{1} \\
\Omega_{2} \\
\Omega_{3}
\end{array}\right] \times\left[\begin{array}{ccc}
J_{x x} & 0 & 0 \\
0 & J_{y y} & 0 \\
0 & 0 & J_{z z}
\end{array}\right]\left[\begin{array}{l}
\Omega_{1} \\
\Omega_{2} \\
\Omega_{3}
\end{array}\right]
$$

Each rotor of the quadrotor has an angular velocity $\omega_{j}$ to define the trust and the drag forces $T_{j}=$ $k_{T} \omega_{j}^{2}$ and $\tau_{j}=k_{m} \omega_{j}^{2}$ respectively, where the $k_{T}$ and $k_{m}$ are the thrust and drag coefficients. The equation for mapping of the roll, pitch, and yaw direction can be written as:

$$
\left[\begin{array}{c}
T \\
M_{1} \\
M_{2} \\
M_{3}
\end{array}\right]=\left[\begin{array}{cccc}
1 & 1 & 1 & 1 \\
0 & -l & 0 & l \\
l & 0 & -l & 0 \\
-c_{\tau} & c_{\tau} & -c_{\tau} & c_{\tau}
\end{array}\right]\left[\begin{array}{c}
T_{1} \\
T_{2} \\
T_{3} \\
T_{4}
\end{array}\right]
$$
becomes:

If we take into account the speeds of the attached propellers to the drivers (motors), the last equation

$$
\left[\begin{array}{c}
T \\
M_{1} \\
M_{2} \\
M_{3}
\end{array}\right]=\left[\begin{array}{cccc}
b & b & b & b \\
0 & -b l & 0 & b l \\
b l & 0 & -b l & 0 \\
-b c_{\tau} & b c_{\tau} & -b c_{\tau} & b c_{\tau}
\end{array}\right]\left[\begin{array}{c}
\omega_{1}^{2} \\
\omega_{2}^{2} \\
\omega_{3}^{2} \\
\omega_{4}^{2}
\end{array}\right]
$$

\section{CONTROL DESIGN}

After developing the dynamic model of the vehicle in the SE(3), The moment vector and the thrust magnitude are the control inputs of our system. The structure of the geometric control law is shown in Figure 2.

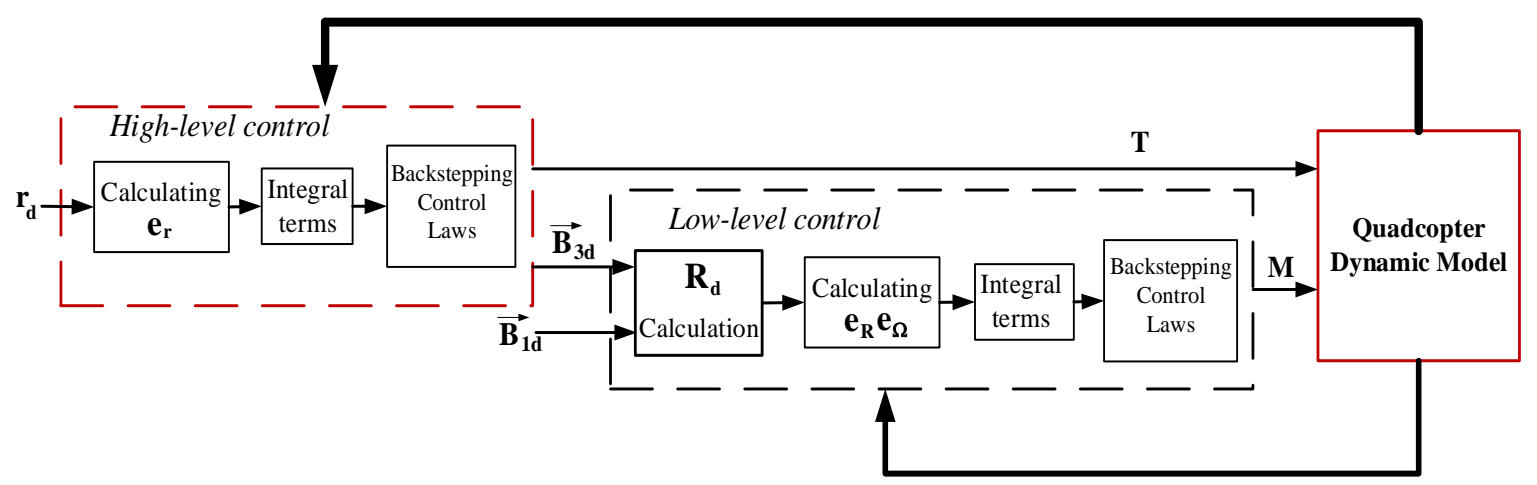

Figure 2. Bloc schematic of the control 
where $T \in \mathbb{R}$ associated with the desired position of the quadrotor $r_{d}$, the moment control is associated with the desired direction of the first body-fixed frame $\vec{b}_{1 d}$ and the desired direction of the third body-fixed frame $\vec{b}_{3 d}$ [21-23]. Thus, the desired position is $r_{d}$, wherein this case, we can obtain the corresponding desired attitude $R_{d}$ :

$$
R_{d}=\left[\vec{R}_{1 d}, \vec{R}_{2 d}, \vec{R}_{3 d}\right] \in S O(3)
$$

where $\vec{R}_{1 d}=\vec{b}_{2 d} \times \vec{b}_{3 d}, \vec{R}_{2 d}=\vec{b}_{2 d}=\frac{\vec{b}_{3 d} \times \vec{b}_{1 d}}{\left\|\vec{b}_{3 d} \times \vec{b}_{1 d}\right\|}$ and $R_{3 d} \overrightarrow{=b}_{3 d}$ $\mathbb{R}^{3}$

From the attitude kinematics represented by (2a) and (6), we obtain angular velocity command $\Omega_{d} \in$

\subsection{High-level position control}

In this part, we use backstepping control to deduce the thrust magnitude $T \in \mathbb{R}$ and the desired direction of the third body-fixed frame $\vec{b}_{3 d}$. Let's define the position error as $e_{1}=r-r_{d}$ and its derivative $\dot{e}_{1}=\dot{r}-\dot{r}_{d}$. Then, designing the $\xi$ to be as the virtual input and $e_{2}=\dot{r}-\xi$ to be the virtual control error, we select the Lyapunov function $V_{1}$ and its derivative as follow:

$$
\begin{aligned}
& V_{1}=\frac{1}{2} e_{1}^{T} I e_{1} \\
& \dot{V}_{1}=e_{1}^{T} I \dot{e}_{1}=e_{1}^{T} I\left(\dot{r}-\dot{r}_{d}\right)=e_{1}^{T} I\left(e_{2}+\xi-\dot{r}_{d}\right)
\end{aligned}
$$

To make $e_{1}$ progressive stable, the (8) needs to content $\dot{V}_{1}<0$. Then, we select $\xi=\dot{r}-k_{1} e_{1}$, where $k_{1}$ is a positive constant.

$$
\dot{V}_{1}=e_{1}^{T} I\left(e_{2}+\dot{r}_{d}-k_{1} e_{1}-\dot{r}_{d}\right)=e_{1}^{T} I e_{2}-k_{1} e_{1}^{T} I e_{1}
$$

where $e_{2}=\dot{r}-\xi=\dot{r}-\dot{r}_{d}+k_{1} e_{1}$. Because of $e_{2}$ is not generally a zero, so the second step of backstepping is given by $V_{2}$ and its derivative:

$$
\begin{aligned}
& V_{2}=\frac{1}{2} e_{1}^{T} I e_{1}+\frac{1}{2} e_{2}^{T} I e_{2} \\
& \dot{V}_{2}=e_{2}^{T} I \dot{e}_{2}+e_{1}^{T} I \dot{e}_{1}=e_{2}^{T} I\left(\ddot{r}-\ddot{r}_{d}+k_{1} \dot{e}_{1}\right)+\dot{V}_{1} \\
& =e_{2}^{T} I\left(\ddot{r}-\ddot{r}_{d}+k_{1} \dot{e}_{1}\right)-k_{1} e_{1}^{T} I e_{1}+e_{1}^{T} I e_{2} \\
& =e_{2}^{T}\left(g i_{3}-\frac{1}{m} T R i_{3}-\ddot{r}_{d}+k_{1} \dot{e}_{1}\right)-k_{1} e_{1}^{T} I e_{1}+e_{1}^{T} I e_{2}
\end{aligned}
$$

Then, the control law $T$ is designed as follow:

$$
T=\left(-m g i_{3}+m \ddot{r}_{d}+\left(k_{1}^{2}-1\right) e_{1}+\left(k_{1}+k_{2}\right) e_{2}\right) R i_{3}
$$

where $k_{1}$ and $k_{2}$ are positive constants. The Lyapunov function's derivative is negative [24], so in this time, we say that global stability is according and it can control the position $r$ to be progressive stability by the control $T$. After the simulation is done, it becomes clear that we need to add integration, the control $T$ became:

$$
T=\left(\begin{array}{c}
m g i_{3}-m \ddot{r}_{d}+k_{i} \int e_{1}+\left(k_{1}^{2}-1\right) e_{1} \\
+\left(k_{1}+k_{2}\right) e_{2}
\end{array}\right) R i_{3}
$$

\subsection{Low-level attitude control}

The backstepping geometric controller uses an error function while using the backstepping control approach to improve its robustness. We notice that it is not possible to compare directly between $R$ and $R_{d}$ due to the tangent vector of $\dot{R}$ and $\dot{R_{d}}$ which are in different tangent spaces.

In (14) defines the attitude error, and (15) defines the Angular Velocity error: 


$$
e_{R}=\frac{1}{2}\left(R_{d}^{T} R-R^{T} R_{d}\right)^{v}
$$

where $\circ^{v}$ is vee map $\left(\circ^{v}: S O(3) \rightarrow \mathbb{R}^{3}\right.$ is the inverse of ${ }^{\circ} \times$ which was defined before.

$$
e_{\Omega}=\Omega-R^{T} R_{d} \Omega_{d}
$$

However, before obtaining the attitude controller dynamics, we need to know $\dot{e}_{R}$ and $\dot{e}_{\Omega}$, which are defined by (16) and (17):

$$
\begin{aligned}
& \left.\dot{e}_{R}=\frac{1}{2}\left(\operatorname{tr}\left(R_{d}^{T} R\right)^{T} I\right]\right) e_{\Omega}=D e_{\Omega} \\
& \dot{e}_{\Omega}=\dot{\Omega}+\left(\Omega_{x} R_{d}^{T} R-R_{d}^{T} R \dot{\Omega}_{d}\right)
\end{aligned}
$$

The matrix $D$ defined in (17) is invertible when the rotation angle between $R$ and $R d$ is less than $180^{\circ}$. To control the position of our quadrotor and the attitude implicitly, we use the following positive Lyapunov function:

$$
V_{3}=\frac{1}{2} e_{R}^{T} I e_{R}+\frac{1}{2} e_{\Omega}^{T} J e_{\Omega}
$$

The derivative of the Lyapunov function is given as:

$$
\begin{aligned}
& \dot{V}_{3}=e_{\Omega}^{T} J\left(\dot{\Omega}+\left(\Omega_{x} R_{d}^{T} R-R_{d}^{T} R \dot{\Omega}_{d}\right)\right)+e_{R}^{T} I \dot{e}_{R} \\
& \dot{V}_{3}=e_{\Omega}^{T}\left((M-\Omega \times J \Omega)+J\left(\Omega_{x} R_{d}^{T} R-R_{d}^{T} R \dot{\Omega}_{d}\right)\right)+e_{R}^{T} I \dot{e}_{R}
\end{aligned}
$$

The control law becomes:

$$
M=-k_{3} e_{R}-k_{5} e_{\Omega}+\Omega \times J \Omega-\mathrm{J}\left(\Omega_{\times} R^{T} R_{d} \Omega_{d}-R^{T} R_{d} \dot{\Omega}_{d}\right)
$$

Concerning the attitude error, we add an integrator controller to the control law $M$ :

$$
M=-k_{3} e_{R}-k_{4} \int e_{R} d t-k_{5} e_{\Omega}+\Omega \times J \Omega-\mathrm{J}\left(\Omega_{\times} R^{T} R_{d} \Omega_{d}-R^{T} R_{d} \dot{\Omega}_{d}\right)
$$

where $k_{3}, k_{4}$ and $k_{5}$ are positive constants, which can be calculated approximately as demonstrated in the stability analysis section as follow:

- Among the properties of these errors, we quote:

$$
\frac{\left\|\dot{e}_{R}\right\|}{2} \leq \rho\left(R, R_{d}\right) \leq \frac{1}{\alpha-1}\left\|e_{R}^{2}\right\|
$$

- The time derive of $\rho\left(R, R_{d}\right)$ is given by:

$$
\dot{\rho}\left(R, R_{d}\right)=e_{R} e_{\Omega}
$$

- We have [25, 26]:

$$
\left\|\dot{e}_{R}\right\|<\left\|e_{\Omega}\right\|
$$

According to the relation of (25), we can write:

$$
\begin{aligned}
& z_{1}^{T} M_{11} z_{1}+k_{4}\left\|\int e_{R} d t\right\| .\left\|e_{\mathrm{R}}+\frac{1}{2} \int e_{R} d t\right\| \leq V \leq z_{1}^{T} M_{12} z_{1}+ \\
& k_{4}\left\|\int e_{R} d t\right\| \cdot\left\|e_{\mathrm{R}}+\frac{1}{2} \int e_{R} d t\right\|
\end{aligned}
$$

Where, 
$z_{1}^{T}=\left[\begin{array}{ll}\left\|e_{\mathrm{R}}\right\| & \left\|e_{\Omega}\right\|\end{array}\right], M_{11}=\frac{1}{2}\left[\begin{array}{cc}k_{3}+k_{4} & \lambda_{M}(J) \\ \lambda_{M}(J) & \lambda_{m}(J)\end{array}\right], M_{12}=\frac{1}{2}\left[\begin{array}{cc}\frac{2\left(k_{3}+k_{4}\right)}{2-\alpha} & \lambda_{M}(J) \\ \lambda_{M}(J) & \lambda_{M}(J)\end{array}\right], \lambda_{m}(J)$ and $\lambda_{M}(J)$ represent the minimum and maximum eigenvalues of the matrix $J$, respectively.

Even to be the derive of Lyapunov function is a negative define should check that relation:

$$
\dot{V}<-z_{1}^{T} L_{1} Z_{1}
$$

where:

$$
L_{1}=\left[\begin{array}{cc}
\left(k_{3}-k_{4}\right. & 0 \\
0 & k_{3}-\lambda_{m}(J)
\end{array}\right]
$$

The matrices $M_{11}, M_{11}$ and $L_{1}$ must be defined positive with the following conditions:

$$
\left\{\begin{array}{c}
k_{4}<k_{3} \\
k_{3}<\min \left\{\frac{\lambda_{M}^{2}(J)}{\lambda_{m}(J)}, \frac{\lambda_{M}(J)}{2}(\alpha-2)\right\} \\
k_{5}>\operatorname{Max}\left\{\frac{\lambda_{M}^{2}(J)}{\lambda_{m}(J)}-k_{3}, \frac{\lambda_{M}(J)}{2}(\alpha-2)-k_{3}, \lambda_{M}(J)\right\}
\end{array}\right.
$$

The formula of $\vec{b}_{3 d}$ became

$$
\vec{b}_{3 d}=-\frac{\left(m g i_{3}-m \ddot{r}_{d}+k_{i} \int e_{1}+\left(k_{1}^{2}-1\right) e_{1+}\left(k_{1}+k_{2}\right) e_{2}\right)}{\left\|m g i_{3}-m \ddot{r}_{d}-k_{i} \int e_{1}-\left(k_{1}^{2}-1\right) e_{1}-\left(k_{1}+k_{2}\right) e_{2}\right\|}
$$

where we assume that

$$
m g i_{3}-m \ddot{r}_{d}-k_{i} \int e_{1}-\left(k_{1}^{2}-1\right) e_{1}-\left(k_{1}+k_{2}\right) e_{2} \neq 0
$$

The controllers are based on a Backstepping geometric method. This control is a cascade where we used control backstepping for the position, and attitude tracking with the need for the tow desired values $r_{d}$ and $b_{1 d}$.

\section{SIMULATION RESULTS}

In this section, we define the quadrotor's parameter values, as shown in Table 1 . Then, we specify the desired input.

Table 1. The parameters of quadrotor UAV

\begin{tabular}{ccc}
\hline Point Size & Purpose in Paper & Special Appearance \\
\hline Mass & $m$ & $4.34 \mathrm{~kg}$ \\
thrust coefficient & $b$ & $3.3 .10^{-5} \mathrm{~N} / \mathrm{rad} / \mathrm{s}$ \\
drag coefficient & $c_{\tau}$ & $0.0226 \mathrm{~m}$ \\
longer & $l$ & $0.314 \mathrm{~m}$ \\
Inertia x & $j_{x x}$ & $0.082 \mathrm{kgm}^{2}$ \\
Inertia y & $j_{y y}$ & $0.082 \mathrm{kgm}^{2}$ \\
Inertia z & $j_{z z}$ & $0.1377 \mathrm{kgm}^{2}$ \\
Maximum speed & Max.rpm & $1300 \mathrm{rpm}$ \\
\hline
\end{tabular}

The initial hovering conditions are: $r(0)=\Omega(0)=[0,0,0]^{T}, R(0)=I_{3 \times 3}$

The desired trajectory $r_{d}=\left[r_{1 d}, r_{2 d}, r_{3 d}\right]^{T}$ varies as follows:

First, the quadrotor takes off vertically to $15 \mathrm{~m}$. Then, the desired direction of the first body-fixed frame $\vec{b}_{1 d}$ takes $5 \mathrm{~m}$ at $\mathrm{t}=2 \mathrm{~s}$, while at time equal $4 \mathrm{~s}$ the quadrotor's coordinate becomes $[5,10,-15]$. At $\mathrm{t}=$ $8 \mathrm{~s}$, the desired direction of the second body-fixed frame $\vec{b}_{2 d}$ decreases to $3 \mathrm{~m}$ and the $\vec{b}_{1 d}$ decreases to $8 \mathrm{~m}$ at $\mathrm{t}$ $=10 \mathrm{~s}$. 
In the second case, we design robust control by changing the inertia by $100 \%$ in the initial $J=1.5 *$ $\left[j_{x x}, j_{y y}, j_{z z}\right]$ and $m=2 * m$. In the third section, we apply an external force that we consider, such as wind gust $F_{e x}=[20,30,0] N$. The first force (20) at time $t \geq 2 s$ and the second force (30) at $t \geq 6 s$.

Simulation results of Figure 3(a) on the right side show the position of the components in three directions, which are almost the same as the desired values, and on the left side show its error, which is defined as $e_{1}=r-r_{d}$. The components in the three directions converge to the desired states or stable states. We observed the position of $\mathrm{x}$, where it converges to the desired trajectory at $\mathrm{t}=1.5 \mathrm{~s}$, y converges at $\mathrm{t}=1 \mathrm{~s}$, while z converges in almost $2.5 \mathrm{~s}$. Figure 3(b) clearly shows the input control $T$ and $M$, where the thrust was between $\mp 200 N$. After $12 \mathrm{~s}, M 2$ and $M 3$ become zero. Figure 3(c) shows the third body-fixed frame $\vec{b}_{3}$ without changing the parameters. Figure 3(d) shows the phase plan of Euler angles, which presents the clear stability of the system. Figure 4(a) shows the results of the position of the third body-fixed frame $\vec{b}_{3}$ after changing the mass and the inertia, and Figure 4(b) then an external force $F_{\text {ex }}$ was applied. We observed the suggested control robustness. Figure (5) shows the trajectory of the quadrotor in 3D for two cases before and after changing the mass and the inertia by applying an external force $F_{e x}$.
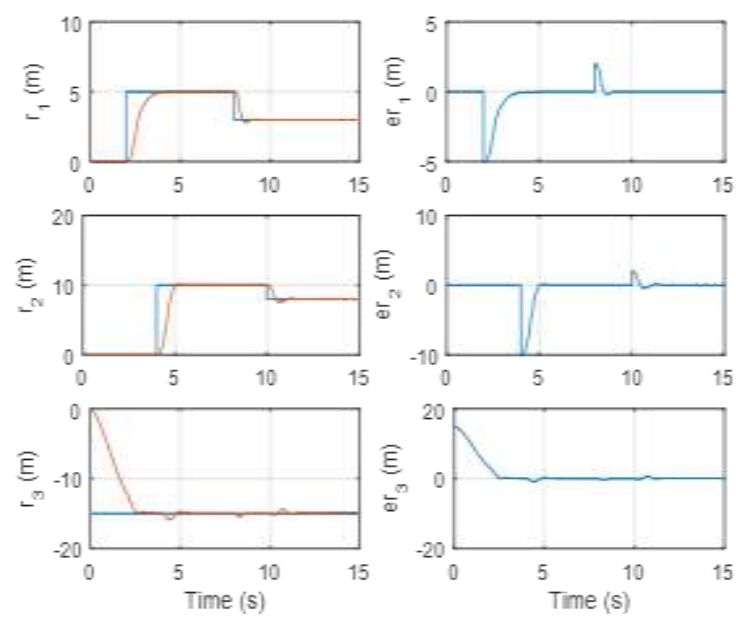

(a) Position and its error
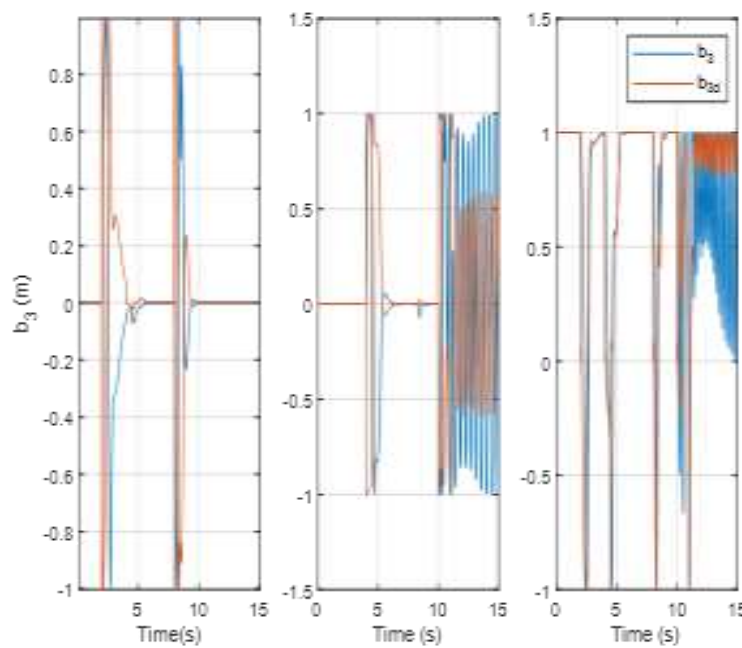

(c) The desired direction of the third body-fixed frame $\vec{b}_{3 d}$
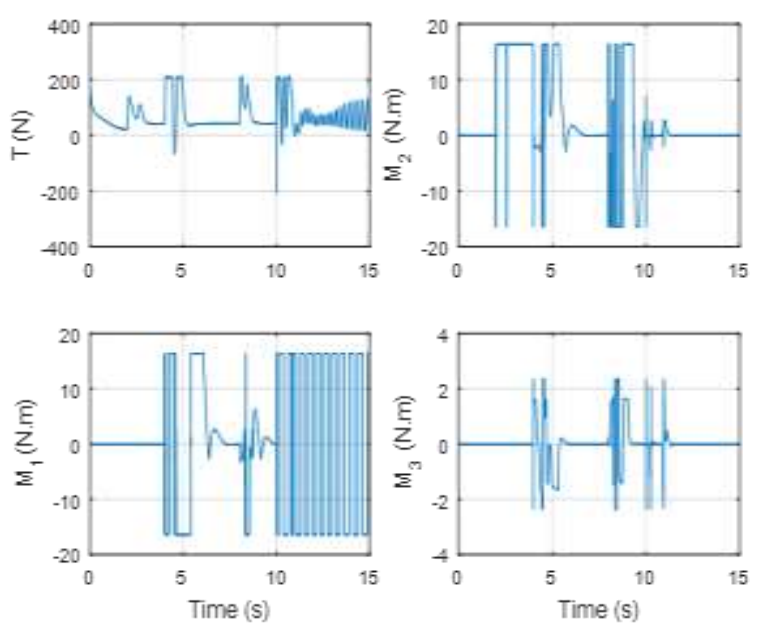

(b) Thrust and different moments
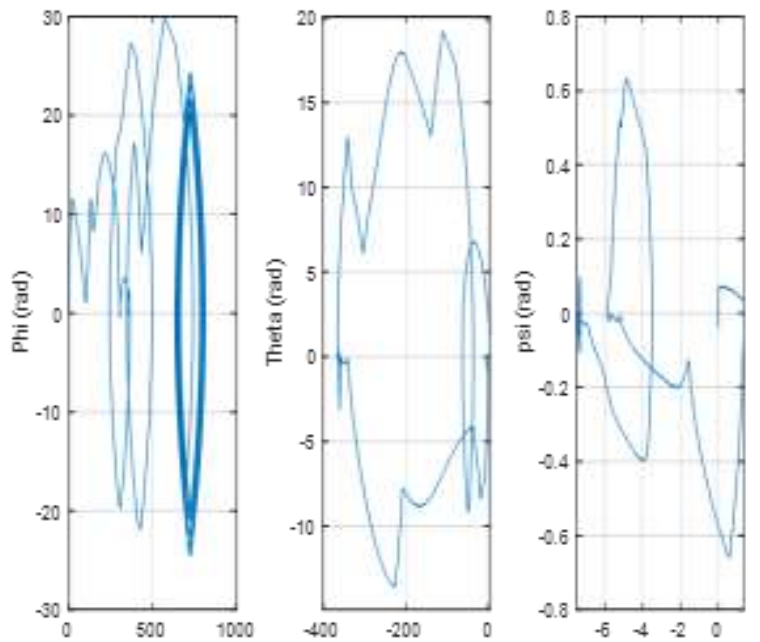

(d) Phase plan of Euler anglers

Figure 3. Flipping using backstepping control without changing the parameters 

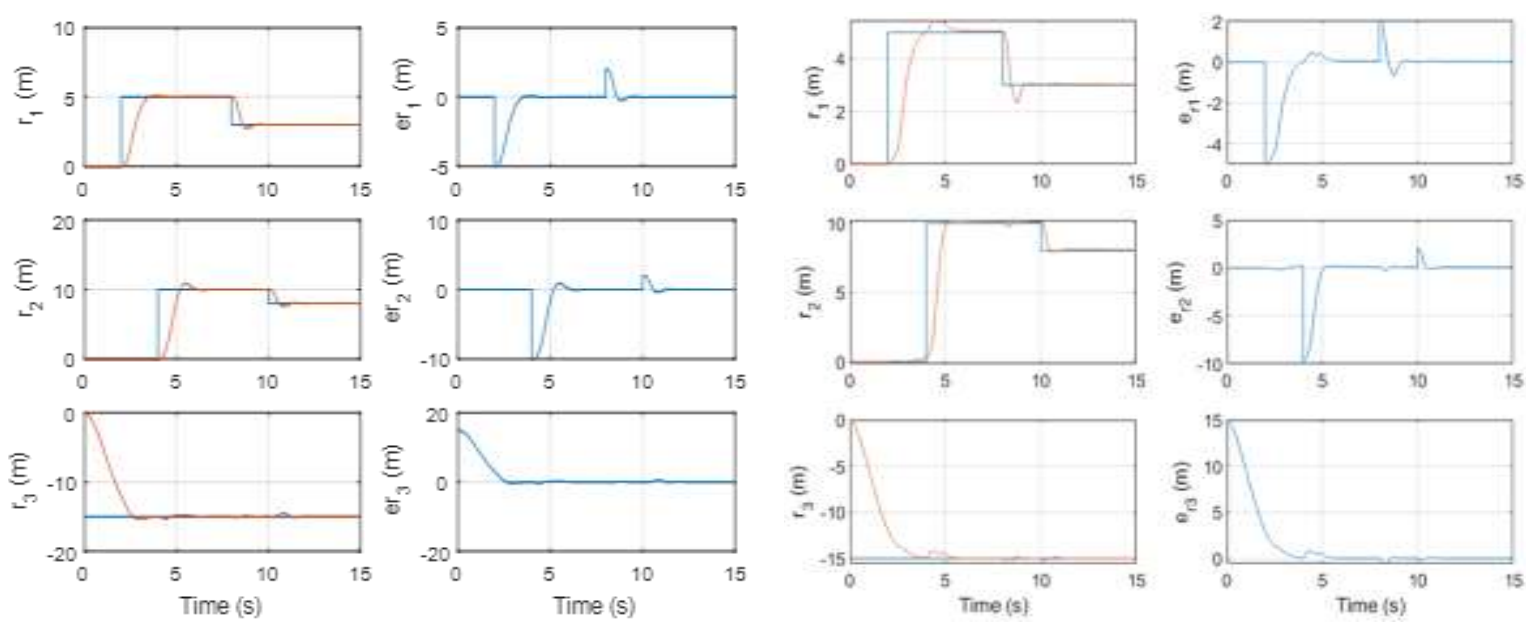

(a) Trajectory with changing the parameters

(b) Trajectory with an external force

Figure 4. Flipping using backstepping control with disturbance

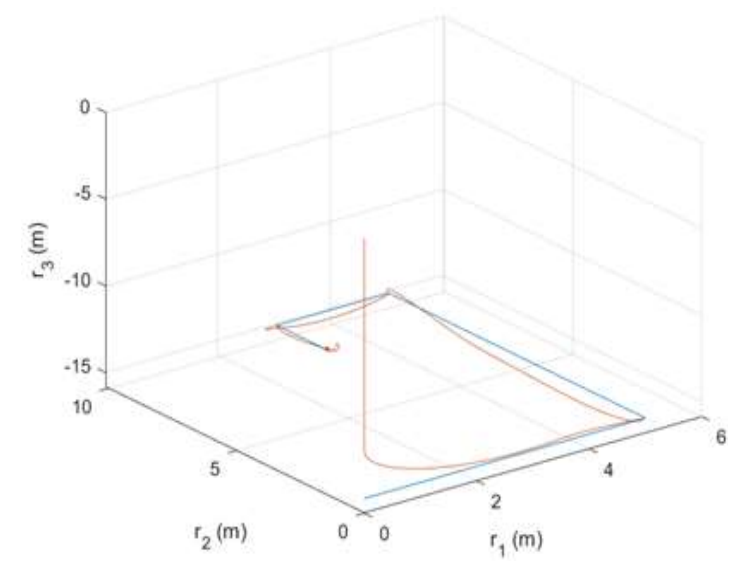

(a) Trajectory without an external force

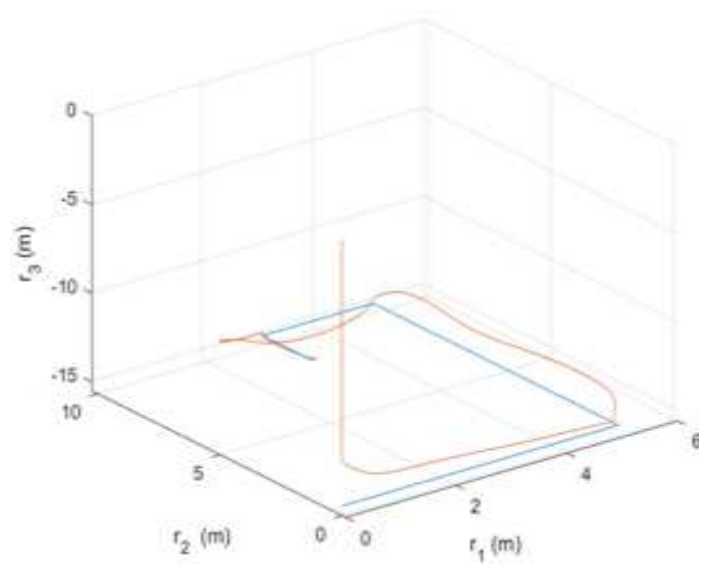

(b) Trajectory with an external force

Figure 5. Global trajectory of the quadrotor in 3 dimensions

\section{CONCLUSION}

In this work, a dynamic global model for a quadrotor UAV was presented. Furthermore, a new tracking controller's development was designed based on a backstepping geometric method developed directly on the $\mathrm{SE}(3)$ to avoid singularities and ambiguities inherent to other attitude representations. The main conclusions are summarized as follows, (a) The state variables can converge fast to the desired value. (b) The errors of position and attitude angles can quickly converge to 0 or stay in a small percentage. (c) The new control strategy which suits complex flight missions can make the quadrotor track different trajectories. Thus, simulations have validated the different prescribed trajectories tracked by actual trajectories very well with this paper's proposed method. Future work should be focused on designing a robust trajectory tracking method that can be applied to complex models and improve the controller's stabilization.

\section{REFERENCES}

[1] Nur Achmad Sulistyo Putro, Andi Dharmawan, Tri Kuntoro Priyambodo, "Quadrotor Control System with Hand Movement Sign as an Alternative Remote Control," International Journal of Robotics and Automation (IJRA), vol. 6, no. 2, pp. 131-140, 2017, doi: 10.11591/ijra.v6i2.pp131-140.

[2] J. Yu, C. Yan and M. Huang, "Research of consistency problem for quadrotor UAV system with leader-follower," 2019 Chinese Automation Congress (CAC), Hangzhou, China, 2019, pp. 616-621, doi: 10.1109/CAC48633.2019.8996473.

[3] Lasmadi Lasmadi, Adha Imam Cahyadi, Samiadji Herdjunanto, Risanuri Hidayat, "Inertial Navigation for Quadrotor Using Kalman Filter with Drift Compensation," International Journal of Electrical and Computer Engineering (IJECE), vol. 7, no. 5, pp. 2596-2604, 2017, doi: 10.11591/ijece.v7i5.pp2596-2604. 
[4] S. Li, N. Duan, Z. Xu and X. Liu, "Tracking Control of Quadrotor UAV with Input Delay," 39th Chinese Control Conference (CCC), Shenyang, China, 2020, pp. 646-649, doi: 10.23919/CCC50068.2020.9188449.

[5] Al Al, Arfita Yuana Dewi, Antonov Bachtiar, Dwi Harinita, "An angle speed and thrust relationship of the rotor system in the quadcopter aircraft," Indonesian Journal of Electrical Engineering and Computer Science (IJEECS), vol. 12, no. 2, pp. 469-474, 2019, doi: 10.11591/ijeecs.v13.i2.pp469-474.

[6] L. Jinyong, Z. Jun, D. Wenjie, G. Xiaoying and Q. Chuntang, "Flying Control System Architecture of Informationization for Pervasive Computing," First International Conference on Pervasive Computing, Signal Processing and Applications, Harbin, 2010, pp. 348-351, doi: 10.1109/PCSPA.2010.91.

[7] F. A. Goodarzi and T. Lee, "Extended Kalman filter on SE(3) for geometric control of a quadrotor UAV," International Conference on Unmanned Aircraft Systems (ICUAS), Arlington, VA, 2016, pp. 1371-1380, doi: 10.1109/ICUAS.2016.7502682.

[8] T. Tan, J. Yin and H. Zhou, "High Precision Attitude Control of Space Maneuvering Platform Based on Model Predictive and Inversive Method*," International Conference on Unmanned Systems (ICUS), Beijing, China, 2019, pp. 340-347, doi: 10.1109/ICUS48101.2019.8995999.

[9] F. Goodarzi, D. Lee and T. Lee, "Geometric nonlinear PID control of a quadrotor UAV on SE(3)," 2013 European Control Conference (ECC), Zurich, 2013, pp. 3845-3850, doi: 10.23919/ECC.2013.6669644.

[10] S. Zhang and C. Chen, "Backstepping Based Nonlinear Integral Sliding Mode Control for Quadrotors Under External Disturbances," Chinese Control Conference (CCC), Guangzhou, China, 2019, pp. 8355-8359, doi: 10.23919/ChiCC.2019.8865215.

[11] L. Zhou and B. Zhang, "Quadrotor UAV Flight Control Using Backstepping Adaptive Controller," 6th International Conference on Control Science and Systems Engineering (ICCSSE), Beijing, China, 2020, pp. 163 166, doi: 10.1109/ICCSSE50399.2020.9171967.

[12] O. Y. Voronkov, "Synergetic Synthesis of the Hierarchical Control System of the "Flying Platform"," International Conference on Control in Technical Systems (CTS), St. Petersburg, Russia, 2019, pp. 23-26, doi: 10.18522/23113103-2020-3-29-39.

[13] Y. Zou, Y. Yin and J. Song, "Flight control of a flying-wing UAV based on active disturbance rejection control," 3rd International Conference on Control Science and Systems Engineering (ICCSSE), Beijing, 2017, pp. 50-55, doi: 10.1109/CCSSE.2017.8087893.

[14] M. Hu, J. Song and M. Yang, "Sliding mode control and control allocation for Electromagnetic Formation Flying," 35th Chinese Control Conference (CCC), Chengdu, 2016, pp. 5810-5813, doi: 10.1109/ChiCC.2016.7554265.

[15] Almido H Ginting, Oyas Wahyunggoro, Adha Imam Cahyadi, "Attitude Control of Quadrotor Using PD Plus Feedforward controller on SO(3)," International Journal of Electrical and Computer Engineering (IJECE), vol. 8, no. 1, pp. 566-577, 2018, doi: 10.11591/ijece.v8i1.pp566-575.

[16] X. Ji, J. Li and Y. Niu, "Optimal attitude control of a rigid body on special orthogonal group using LGVI and FDGMRES," 20th International Conference on Methods and Models in Automation and Robotics (MMAR), Miedzyzdroje, 2015, pp. 93-98, doi: 10.1109/MMAR.2015.7283853.

[17] T. -y. Chen and J. -t. Yu, "Quadrotor Attitude Control Using Special Orthogonal Matrix," International Automatic Control Conference (CACS), Hsinchu, 2020, pp. 1-5, doi: 10.1109/CACS50047.2020.9289762.

[18] W. Chenlu, C. Zengqiang, S. Qinglin and Z. Qing, "Design of PID and ADRC based quadrotor helicopter control system," Chinese Control and Decision Conference (CCDC), Yinchuan, 2016, pp. 5860-5865, doi: 10.1109/CCDC.2016.7532046.

[19] L. Jiajin, L. Rui, S. Yingjing and Z. Jianxiao, "Design of attitude controller using explicit model predictive control for an unmanned quadrotor helicopter," Chinese Automation Congress (CAC), Jinan, 2017, pp. 2853-2857, doi: 10.1109/CAC.2017.8243262.

[20] Q. Jiao, J. Liu, Y. Zhang and W. Lian, "Analysis and design the controller for quadrotors based on PID control method," 33rd Youth Academic Annual Conference of Chinese Association of Automation (YAC), Nanjing, 2018, pp. 88-92, doi: 10.1109/YAC.2018.8406352.

[21] X. Zhang, Y. Fang, X. Zhang, J. Jiang and X. Chen, "A Novel Geometric Hierarchical Approach for Dynamic Visual Servoing of Quadrotors," in IEEE Transactions on Industrial Electronics, vol. 67, no. 5, pp. 3840-3849, May 2020, doi: 10.1109/TIE.2019.2917420.

[22] J. Liu and G. Lee, "A Carrot in Probabilistic Grid Approach for Quadrotor Line Following on Vertical Surfaces," International Conference on Unmanned Aircraft Systems (ICUAS), Atlanta, GA, USA, 2019, pp. 1234-1241, doi: 10.1109/ICUAS.2019.8797792.

[23] T. Lee, K. Sreenath and V. Kumar, "Geometric control of cooperating multiple quadrotor UAVs with a suspended payload," 52nd IEEE Conference on Decision and Control, Florence, 2013, pp. 5510-5515, doi: 10.1109/CDC.2013.6760757.

[24] Danh Huy Nguyen, Tung Lam Nguyen, Manh Linh Nguyen, Huy Phuong Nguyen, "Nonlinear Control of an Active Magnetic Bearing with Output Constraint," International Journal of Electrical and Computer Engineering (IJECE), vol. 8, no. 5, pp. 3666-3677, 2018, doi: 10.11591/ijece.v8i5.pp3666-3677.

[25] T. Lee, M. Leok and N. H. McClamroch, " Geometric tracking control of a quadrotor UAV for extreme maneuverability," IFAC Proceedings, vol. 44, no. 1, 2011, pp. 6337-6342.

[26] F. A. Goodarzi, D. Lee and T. Lee, "Geometric stabilization of a quadrotor UAV with a payload connected by flexible cable," 2014 American Control Conference, Portland, OR, 2014, pp. 4925-4930, doi: 10.1109/ACC.2014.6859419. 